\title{
Development and Evaluation of an Instrument to Measure Health-Related Quality of Life in Cuban Breast Cancer Patients Receiving Radiotherapy
}

\author{
Josefina Lugo MD, Misleidy Nápoles MD, Inés Pérez MD, Niurka Ordaz, Mario Luzardo PhD, Leticia Fernández DDS PhD
}

\begin{abstract}
INTRODUCTION Although modern technology has extended the survival of breast cancer patients, treatment's adverse effects impact their health-related quality of life. Currently, no instrument exists capable of identifying the range of problems affecting breast cancer patients receiving radiotherapy in Cuba's socioeconomic and cultural context.

OBJECTIVES Construct and validate an instrument to measure the effects of breast cancer and radiotherapy on health-related quality of life in Cuban patients.

METHODS The study was conducted at the Oncology and Radiobiology Institute, Havana, Cuba, from January 2010 through December 2011. Inclusion criteria were: adult female, histological diagnosis of breast cancer, treated with ambulatory radiotherapy, and written informed consent; patients unable to communicate orally or in writing, or who had neurologic or psychiatric conditions were excluded. Development phase: focus groups guided by a list of questions were carried out with 50 women. The patients reported 61 problems affecting their health-related quality-of-life. A nominal group (six oncologists and two nurses) identified the same problems. A syntactic analysis of the information was performed to create items for study and measurement scales. Content validity was determined by a nominal group of seven experts using professional judgment. Another 20 patients were selected to evaluate face validity. Validation phase: the instrument
\end{abstract}

\section{INTRODUCTION}

Technological developments in oncology have resulted in new treatments with great curative potential. However, while survival times have increased, corresponding benefits for health-related quality of life (HRQOL) have not been achieved. Thus, the literature reflects increasing interest in adding measurements of $\mathrm{HRQOL}$ to biomedical variables assessed in clinical trials of cancer therapies.[1-3]

Breast cancer and its treatment have negative effects on patients' HRQOL. Besides fear of mastectomy and the anxiety and uncertainty about chemotherapy cytotoxicity, many patients worry that daily radiotherapy over several weeks may cause radiotoxicity, provoking temporary interruption of therapy. This period, and the post-treatment phase, while waiting for tumor response, challenge patients' coping capacities. Some patients are not prepared to face these problems-physically, psychologically or socially.[4-12]

HRQOL is multidimensional and essentially subjective. Despite all research efforts, it has no standard definition.[1316] Various authors suggest that HRQOL covers four essential domains: physical wellbeing (autonomy and physical capacity), somatic discomfort (symptoms related to disease and/or treatment), psychological state (emotions, anxiety, depression), and problems with social relations (familial and professional). [17-20] was applied to 230 patients at three different points: before radiotherapy, at the end of radiotherapy and four weeks after radiotherapy was concluded. Reliability, construct validity, discriminant validity, predictive validity, interpretability and response burden were evaluated.

RESULTS The final instrument developed had 33 items distributed in 4 domains: physical functioning, psychological functioning, social and family relationships, and physical and emotional adverse effects of disease and treatment. There were two discrete items: perceived general health and perceived health-related quality of life. Content validity and face validity were assessed as acceptable, by experts and patients respectively. Homogeneity, construct validity, and discriminant validity were satisfactory. The best results were obtained with test-retest reliability, predictive validity, and interpretability; the low rate of unanswered questions indicated that the instrument did not produce excessive patient response burden.

CONCLUSION The new instrument fulfilled the requirements for measuring impact of breast cancer and of radiotherapy on health-related quality of life in these Cuban patients, validating its usefulness for inclusion in clinical trial protocols.

KEYWORDS Psychometrics, quality of life, breast cancer, radiotherapy, Cuba

Our literature review found general agreement regarding the influence of culture on wellbeing and health. Siegrist found differences in effectiveness and perceived effectiveness of medical treatments in different cultural contexts,[21] and Grossi found several cultural variables to be important determinants of individual psychological wellbeing.[22]

Most instruments to measure HRQOL have been created in English; some others, less used, were written in French or German. $[23,24]$ But a simple translation is not enough for an instrument's applicability outside the country where it was developed, because more important than language are conceptual and cultural similarities.[25,26] An instrument will retain traces of cultural characteristics from its country of origin, and these will be more obvious in proportion to the sociocultural and language differences of the country where it is to be adapted.

The traditional philosophy of psychometric instrument construction assumes that the instrument's set of items (indicator variables) reflects unobservable latent constructs intended for measurement, indicating their level or degree (e.g. of depression, intelligence, etc.). In developing instruments to measure the HRQOL construct, two kinds of items are frequently used: indicator variables, which concern the level of HRQOL (psychological functioning and social/family relations) and causal variables, which refers to factors such as symptoms and adverse effects of treatment that produce changes in HRQOL (physical 
functioning, disease symptoms and adverse effects of cancer treatment).[27,28]

Of prime importance in an instrument that includes causal variables are basic properties such as test-retest reliability, content validity, discriminant validity (ability to detect differences between groups known to be different), predictive validity (sensitivity to changes over time) and clinical interpretability. Also needed are simplicity, understandability by the patient and applicability within the socioeconomic and cultural context of the population studied.

Thus, measuring HRQOL in Cuban breast cancer patients receiving radiotherapy requires an instrument developed in the Cuban socioeconomic and cultural environment. This will provide information about problems distressing patients from their own perspectives, since they know their own emotions best. As a result, when deciding appropriate therapies, patient needs could be more fully considered: subjective morbidity (negative emotional states and psychosomatic symptoms) as well as the impact of the disease and its treatment on patient lifestyles. In addition, knowledge derived from the instrument's application would facilitate identification of patients requiring specialized attention to improve their individual and social responses to the real challenges of daily life. The purpose of this research was to construct and validate just such an instrument.

\section{METHODS}

This study was conducted at the Oncology and Radiobiology Institute (INOR, the Spanish acronym) in Havana, in 2010-2011. Participants were selected nonprobabilistically by the following criteria: women aged $>18$ years with histological diagnosis of breast cancer, referred for ambulatory radiotherapy, who provided written informed consent for participation. Excluded were patients unable to communicate orally or in writing, and patients with mental retardation, psychopathological symptoms, senile dementia or cerebral metastasis. The work was done in two phases: development and validation of the instrument.

Development phase The different items were generated by a qualitative method after collecting testimonies from patients attending their last session of ambulatory radiotherapy. Sample size was determined using the saturation of information criterion, interviews stopped when no novel points emerged, that is, when the last five women reported items that had already been included.[29] A total of 50 patients participated in this phase, organized in 10 focus groups of 5 patients each. To keep interviews uniform, a question guide was elaborated, including different aspects of HRQOL documented in the literature.[4-20,30] [A translation of the focus group guide is available online at www.medicc.org/mediccreview/Lugo-Eds.]

All interviews were done in settings providing adequate privacy. To encourage patient spontaneity, no recording equipment was used for the focus group interviews; patients were asked to stay on topic for each question asked.

For analysis and qualitative interpretation of the information gathered, a problem list was created and organized by topic, eliminating those that repeated the same idea and grouping those with similar meaning. This allowed identification of terms that were later included under the same item (e.g., despair and frustration). Problems that decreased HRQOL were documented and sub- mitted for evaluation to a nominal group formed by experts (six oncologists and two nurses), each with $\geq 15$ years of experience treating breast cancer patients.

A preliminary version of the instrument, called CV-MRT-P, was constructed with 42 items (distilled by semantic analysis from the 61 problems identified), using a 5-point Likert-like scale;[31] it described the items clearly and plainly, used positive and negative language, and avoided technical jargon.

An expert group of 5 oncologists, 1 biostatistician, and 1 psychologist, each with $\geq 15$ years of experience in this area, assessed content validity of this preliminary version. Each item was evaluated according to whether it was: 1) important to patients, 2) easy for patients to understand, 3) clearly and explicitly associated with the HRQOL concept to be measured, 4) associated with the domains of the instrument, 5) formulated to be consistent with the question's possible responses, 6) capable of eliciting varying responses among different patients, 7) worded in a way compatible with its operationalization (scale categories equidistant and hierarchically related to the data), and 8) not in violation of ethical principles.

Face validity was assessed by 20 eligible patients not involved in generating the items. They were asked to answer the questions included in the CV-MRT-P and to give their opinion about the instrument's clarity, comprehensibility and simplicity. They were also asked to add any missing issue affecting their HRQOL.

Each answer was given a value from 1 to 5 ; the higher the value, the better the HRQOL. The mean scores for component items in each HRQOL domain were summarized for each corresponding synthetic variable:

- physical functioning (PhF)

- psychological functioning (PsF)

- social and family relations (SR)

- disease symptoms and adverse effects of treatment (AE)

The variable scores were then summed with the score for one discrete variable, perceived general health $(\mathrm{pH})$, and divided by five to create the global HRQOL scale. Perceived HRQOL (pQ) was judged redundant and not included. Thus the scale expression was:

$\mathrm{HRQOL}=(\mathrm{PhF}+\mathrm{PsF}+\mathrm{SR}+\mathrm{AE}+\mathrm{pH}) / 5$

Validation phase CV-MRT-P (with one modification, described in Results) was administered to 230 participants individually at three different times: before radiotherapy, at the end of radiotherapy and 4 weeks later. Information collected was stored in a database using SPSS 19.0. Dimensionality, construct validity, reliability (test-retest repeatability and internal consistency), discriminant validity, predictive validity, interpretability and response burden were evaluated.

Dimensionality Exploratory factor analysis (EFA) and varimax rotation with Kaiser normalization were used to explore instrument structure and to reduce dimensionality, minimizing information loss. Beforehand, it was decided to keep only items with factor loadings $\geq 0.4$ (correlation between them and their domain). Bartlett sphericity test was performed to verify if it was possible to carry out EFA; it showed a significant result $(<0.05)$ and KaiserMeyer-Olkin sample appropriateness of 0.811 , meaning that the data matrix tolerated factor extraction.[32] 
Construct validity was assessed by examining convergent and divergent validity using the multitrait multimethod correlation matrix. This method examines both types of validity using Pearson correlation. Convergent validity is present if correlations among items in the same domain, and between them and the synthetic variable of the domain to which they theoretically belong, are $\geq 0.4$. Divergent validity is present if there is a correlation of $<0.4$ among items of different domains, and between them and the synthetic variables of the domains to which, in theory, they do not belong.[33]

Reliability The Pearson correlation coefficient was used to assess test-retest repeatability and Cronbach alpha for internal consistency.[34]

Discriminant validity ANOVA was applied to each of the HRQOL domains, stratified according to clinical stage of disease, on the assumption that patients in clinical stages III and IV have worse HRQOL than patients in stages I and II (it should be noted that significant differences may be found between stages without a dose-response effect).

Predictive validity is the ability of the instrument to detect changes in HRQOL produced over time by a particular event or intervention. It estimates the magnitude of changes in the scale and its domains by standardized mean response, calculated by dividing the mean change between initial and final time by its SD.[35] Cohen defines changes in standardized mean response as small, $<0.2$; medium, 0.2 to $<0.8$; and large, $\geq 0.8$.[36]

Clinical interpretability is the degree to which quantitative scores for the scale and its domains translate into qualitative clinical meaning concerning HRQOL deterioration.[37] We defined severe deterioration as 1-1.9; moderate, 2-3.9; mild, 4-4.9; and no deterioration, or normal, as 5 .

Response burden was assessed by average time in minutes patients needed to complete the questionnaire, and by nonresponse rate; the latter was considered low if $\leq 3 \%$ and high if $\geq 10 \%$.

The analysis was repeated with a modified version of the scale (CV-MRT-01): nine items were eliminated and three were moved from PhF to AE. Results presented for CV-MRT-P include dimensionality, construct validity, repeatability, internal consistency and response burden. Results presented for CV-MRT-01 include dimensionality, construct validity, test-retest reliability (repeatability and internal consistency), discriminant validity, predictive validity and interpretability. Response burden was not analyzed in this second round because the modified scale was not administered to patients.

Ethical considerations The study was approved by the INOR research ethics committee. Participants gave written informed consent following an explanation of the study's objectives, benefits and risks.

\section{RESULTS}

Participant age range was $30-75$ years (mean 51 years for those involved in generating the items and assessing face validity, 54.3 years for the 230 women who tested the instrument). Breast cancer stage distribution for the latter group was: stage I, $12.8 \%$; II, $39.9 \%$; III, 36.2\%; and IV, $11 \%$. The focus groups selected 61 problems, related to financial difficulties (3), PhF (8), PsF (30), and $\operatorname{AE}(20$, expressed by women who had completed $90 \%$ of radiotherapy). These problems were confirmed by the nominal group without additions. CV-MRT-P contained 42 items, 5 in $\mathrm{PhF}$, 21 in PsF, 3 in SR, 11 in $A E$, and 2 discrete items: $p H$ and $p Q$.

The experts judged content validity favorably, and the 20 patients who assessed the instrument's face validity had no negative comments. They did suggest a change: in accordance with opinions of $95 \%$ of patients, the item, I have emotional conflicts because I am sick was replaced by Since I've been sick, I feel like crying.

In the preliminary version, EFA extracted four main components able to reproduce the correlations between observed variables; they explain $53.7 \%$ of total variance (Table 1). The first factor included all items in the PhF domain and explained the highest percentage of data variance $(27.3 \%, \lambda=6.282)$. The second component explained $11.1 \%$ of variance; it corresponded to $A E$, where EFA located some items that were more closely correlated with $\mathrm{AE}$ than with PsF: trouble sleeping; sadness, despair or frustration; and decreased sexual interest. This domain was therefore renamed physical and emotional adverse effects (of disease and treatment). The correlation matrix showed $9 \mathrm{PsF}$ items that had very low correlations with the rest $(<0.3)$, thus adding little information (avoids looking at herself at the mirror, avoids contact with others, avoids speaking about the disease, avoids sexual relations, avoids being seen by others, avoids going out, feels diminished self esteem, concerned about negative effects of treatment, feels that treatment is not useful anymore). They were therefore eliminated from the revised instrument.

Table 1: Variance explained in patient responses to two versions of an instrument to measure HRQOL in Cuban breast cancer patients receiving radiotherapy

\begin{tabular}{|c|c|c|c|c|}
\hline \multirow{2}{*}{ Component } & & \multicolumn{3}{|c|}{ Principal component extraction } \\
\hline & & \multirow{2}{*}{$\begin{array}{l}\text { Total } \\
6.282\end{array}$} & \multirow{2}{*}{$\begin{array}{r}\begin{array}{c}\text { Variance } \\
\text { explained (\%) }\end{array} \\
27.3\end{array}$} & \multirow{2}{*}{$\begin{array}{l}\text { Cumulative } \\
\text { variance explained } \\
(\%)\end{array}$} \\
\hline \multirow{4}{*}{ CV-MRT-P } & $\mathrm{PhF}$ & & & \\
\hline & $\mathrm{AE}$ & 2.436 & 11.1 & 38.4 \\
\hline & PsF & 1.823 & 8.2 & 46.6 \\
\hline & SR & 1.612 & 7.1 & 53.7 \\
\hline \multirow{4}{*}{ CV-MRT-01 } & $\mathrm{PhF}$ & 7.694 & 27.6 & 27.6 \\
\hline & $\mathrm{AE}$ & 3.541 & 12.1 & 39.7 \\
\hline & PsF & 2.136 & 9.1 & 48.8 \\
\hline & SR & 1.885 & 8.1 & 56.9 \\
\hline
\end{tabular}

$A E:$ physical and emotional adverse effects HRQOL: health-related quality of life

PhF: physical functioning PsF: psychological functioning

$\mathrm{SR}$ : social and family relations

The new version of the instrument (CV-MRT-01) contained 33 items, distributed in 4 domains. Two of these had changes in number of items: three from PsF were moved to AE. The current structure of PSF and AE includes 9 and 14 items, respectively (Table 2 ). EFA for this version extracted four components that explained somewhat more variance than the original (56.9\%). Variance explained by specific components was similar in both versions.

Table 3 displays the multitrait multimethod matrix resulting from calculation of Pearson correlations between synthetic variables and HRQOL scores for CV-MRT-01 at two points (before and on completion of radiotherapy). These numbers show that the syn- 
Table 2: CV-MRT-01, an instrument to measure HRQOL in Cuban breast cancer patients receiving radiotherapy

\begin{tabular}{l|l|}
\hline Domain/item & Scale \\
\hline Physical Functioning (PhF) & \\
\hline Get out of bed, bathe, dress, comb hair (self sufficiency) & Difficulty \\
Perform housework (role) & 5: none \\
Walk several blocks without help & 4: slight \\
Climb stairs (several floors) without help & 3: moderate \\
Continue working as before & 2: great \\
\hline Psychological Functioning (PsF) & 1: extreme \\
\hline Anxiety, nervousness or unrest & \\
Uncertainty about the future & \\
III-tempered or easily irritable & 5: never \\
Wanting to cry & $4:$ seldom \\
Embarrassment about breast appearance & $3:$ sometimes \\
Decreased desire to enjoy what you used to like most & $2:$ often \\
Eagerness to participate in activities with family and friends* & $1:$ always \\
Financial worries &
\end{tabular}

$\begin{array}{ll} & 5: \text { none } \\ \text { Tendency to hide the disease } & 4: \text { slight } \\ & 3: \text { moderate } \\ & 2: \text { great } \\ 1: \text { extreme }\end{array}$

Social and family relations (SR)

Family's support in the way you need it

Partner's support in the way you need it

Friends' support in the way you need it

5: always

4: often

3: moderate

2: some

1: none

Physical and emotional adverse effects of disease and treatment (AE)

5: never

Sadness, despair or frustration

Decreased sexual interest

4: seldom

3: sometimes

2: often

1: always

\begin{tabular}{|c|c|}
\hline $\begin{array}{l}\text { Sleep disturbance } \\
\text { Fatigue, low energy or lack of energy } \\
\text { Malaise } \\
\text { Nausea or vomiting } \\
\text { Loss of appetite } \\
\text { Trouble swallowing } \\
\text { Pain or increased sensitivity in the breast area } \\
\text { Heat or itching in the breast area } \\
\text { Stinging, irritation or burning sensation in the breast area } \\
\text { Breast inflammation or dryness } \\
\text { Swelling or pain in the arm on the same side of the affected breast }\end{array}$ & $\begin{array}{l}\text { 5: none } \\
\text { 4: slight } \\
\text { 3: moderate } \\
\text { 2: great } \\
\text { 1: extreme }\end{array}$ \\
\hline Pain in other parts of the body & $\begin{array}{l}\text { 5: none } \\
\text { 4: slight } \\
\text { 3: moderate } \\
\text { 2: great } \\
\text { 1: unbearable }\end{array}$ \\
\hline \multicolumn{2}{|l|}{ Discrete items } \\
\hline $\begin{array}{l}\text { Perceived general health }(\mathrm{pH}) \\
\text { Perceived health-related quality of life }(p Q)\end{array}$ & $\begin{array}{l}\text { 5: excellent } \\
\text { 4: good } \\
\text { 3: indifferent } \\
\text { 2: poor } \\
\text { 1: very poor }\end{array}$ \\
\hline
\end{tabular}

*coded inversely to reflect sense of wording

HRQOL: health-related quality of life

thetic variables have higher correlations $(\geq 0.4)$ with HRQOL and moderate correlations with the rest of the synthetic variables, indicating acceptable convergent and divergent validity. Pearson correlation analysis found acceptable patterns of convergence and divergence generally, with the following exceptions: appetite loss did not have an acceptable correlation $(r=0.34)$ with its own domain, AE. Also, swelling or pain in the arm on the same side as the affected breast had better convergence $(r=0.57)$ with PhF than with its own domain, AE $(r=0.38)$, and had low correlations (0.23-0.34) with other items in its own domain, except for pain or increased sensitivity in the breast area and pain in other parts of the body ( $r=0.41$ and $r=0.40$ respectively). Heat or itching in the breast area, and pain in other parts of the body showed low convergence with $\mathrm{AE}(r=0.38$ and $r=$ 0.37 respectively).

Test-retest reliability of CV-MRT-01 domains can be seen on the diagonal of Table 3; values range between 0.72 and 0.87, an improvement over CV-MRT-P (for which the range was $0.65-0.78)$. Overall internal consistency of the instrument and its domains was satisfactory for all three measurements (Cronbach alpha 0.748-0.917), and better than for CV-MRT-P (Cronbach alpha 0.721-0.755). The most important items for internal consistency are those that, when eliminated, decrease Cronbach alpha, i.e., make the domain more homogeneous. In this sense, the most important items were: perform housework; continue working as before; fatigue, low energy or lack of energy; malaise; trouble swallowing; breast inflammation or dryness; pain or increased sensitivity in the breast area; stinging irritation or burning sensation in the breast area; sadness, despair or frustration; decreased desire to enjoy what you used to like most; decreased sexual interest; tendency to hide the disease; family's support in the way you need it; friends' support in the way you need it; $p H$; and $p Q$.

Analysis of discriminant validity (or concurrent validity) for CV-MRT-01 confirmed that, at the end of radiotherapy, patients with more advanced stages of the disease suffered slightly greater decrease in levels of physical and psychological functioning than did patients in less advanced stages (values 3.9-4.4). PhF discriminated between stages I, II, III and IV in the second measurement, and PsF discriminated between stages I and IV in the third measurement.

Predictive validity analysis showed that CV-MRT-01 detected medium-to-large changes $(>0.2)$ in standardized-mean response for HRQOL and its domains, including discrete items. Exceptions were SR, for which standardized-mean response remained stable throughout. Substantial changes $(\geq 0.8)$ took place over both periods. There were negative changes observed in PhF and PsF between beginning and end of radiotherapy, but both improved by four weeks after completion of radiotherapy (Table 4).

Table 5 illustrates the clinical interpretability of the scale and its domains. Scores for PhF, AE, PsF and SR, as well as for the two discrete items $(\mathrm{pH}$ and $\mathrm{pQ}$ ) had values $>3$ at the end of radiotherapy and four weeks later, indicating that at worst, radiotherapy produced a slight deterioration in HRQOL. This inference was confirmed by clinical observation, since none of the women exhibited severe secondary reactions to radiotherapy. 
Table 3: Multitrait multimethod matrix and diagonal test-retest reliability of an instrument to measure HRQOL in Cuban breast cancer patients receiving radiotherapy, before and after radiotherapy

\begin{tabular}{l|l|c|c|c|c|r|}
\multirow{2}{*}{ Domain } & \multicolumn{6}{|c|}{$\begin{array}{c}\text { Correlations between synthetic variables } \\
\text { and HRQOL scores }\end{array}$} \\
\cline { 3 - 8 } & & \multicolumn{5}{|c|}{ First measurement } \\
\cline { 3 - 8 } & PhF & 0.78 & 0.62 & 0.62 & 0.35 & 0.68 \\
\hline \multirow{3}{*}{$\begin{array}{l}\text { Second } \\
\text { measurement }\end{array}$} & AE & 0.26 & 0.72 & 0.74 & 0.39 & 0.84 \\
\cline { 3 - 8 } & PsF & 0.33 & 0.40 & 0.83 & 0.90 & 0.81 \\
\cline { 2 - 7 } & SR & 0.22 & 0.45 & 0.55 & 0.87 & 0.70 \\
\cline { 2 - 7 } & HRQOL & 0.76 & 0.80 & 0.59 & 0.88 & 0.80 \\
\hline
\end{tabular}

AE: physical and emotional adverse effects

HRQOL: health-related quality of life PhF: physical functioning

PsF: psychological functioning SR: social and family relations

Table 4: Predictive validity of an instrument to measure HRQOL in Cuban breast cancer patients receiving radiotherapy

\begin{tabular}{|c|c|c|}
\hline Comparison & Domain & SMR \\
\hline \multirow{6}{*}{ Before RT/on completion of RT } & Physical functioning & -0.6 \\
\hline & $\begin{array}{l}\text { Physical and emotional } \\
\text { adverse effects }\end{array}$ & -1.0 \\
\hline & Psychological functioning & -0.4 \\
\hline & Social and family relations & -0.0 \\
\hline & Perceived general health & -0.6 \\
\hline & Perceived HRQOL & -0.7 \\
\hline \multirow{6}{*}{$\begin{array}{l}\text { On completion of RT/four weeks } \\
\text { after completion }\end{array}$} & Physical functioning & 0.6 \\
\hline & $\begin{array}{l}\text { Physical and emotional } \\
\text { adverse effects }\end{array}$ & 0.8 \\
\hline & Psychological functioning & 0.5 \\
\hline & Social and family relations & 0.1 \\
\hline & Perceived general health & 0.6 \\
\hline & Perceived HRQOL & 0.7 \\
\hline
\end{tabular}

SMR: standardized mean response

Table 5: Mean responses for synthetic and discrete variables in instrument to measure HRQOL in Cuban breast cancer patients, before, on completion of and four weeks after radiotherapy

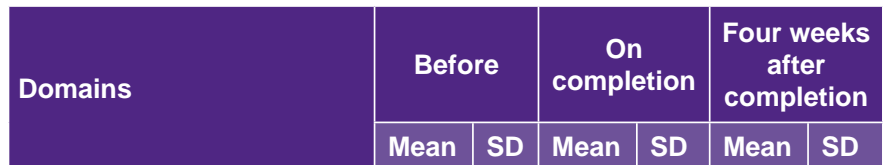

\begin{tabular}{|l|l|l|l|l|l|l|}
\hline Physical functioning & 4.3 & 0.9 & 4.5 & 0.7 & 4.6 & 0.6 \\
\hline $\begin{array}{l}\text { Physical and emotional } \\
\text { adverse effects }\end{array}$ & 4.6 & 0.6 & 4.3 & 0.7 & 4.7 & 0.5 \\
\hline Psychological functioning & 4.5 & 0.7 & 4.6 & 0.6 & 4.7 & 0.5 \\
\hline Social and family relations & 4.8 & 1.0 & 4.6 & 1.2 & 4.7 & 0.9 \\
\hline Perceived general health & 3.8 & 0.7 & 3.8 & 0.6 & 4.0 & 0.5 \\
\hline Perceived HRQOL & 3.9 & 1.1 & 4.0 & 0.9 & 4.2 & 0.7 \\
\hline
\end{tabular}

HRQOL: health-related quality of life

CV-MRT-P did not cause appreciable response burden. Average completion time was 7.2 minutes (4.1-11.2). All items were responded by $100 \%$ of patients, except those for decreased sexual interest, spousal and friends' support, and malaise, all of which had $<10 \%$ nonresponse rates.

\section{DISCUSSION}

A multi-item instrument was constructed, validated and improved; experts and patients agree that it covers all aspects required to evaluate the impact of breast cancer and radiotherapy on Cuban women's HRQOL. The structure of this tool correlates well with the degree of psychological functioning, social and family relations, physical functioning, and physical and emotional adverse effects of disease and treatment, as well as with two discrete items: perception of general health and perception of HRQOL.

For the $\mathrm{PhF}$ and $\mathrm{AE}$ domains, we used clinimetric scales that measure sequence and severity of symptoms and adverse effects of treatment. CV-MRT-01 has 33 items, fewer than both EORTC QLQ-C30 and its complement, BR-23, both of which have 53.[24] It also differs somewhat in structure from these international instruments. For example, there are actually more items in the psychological functioning domain of CV-MRT-01 than in EORTC QLQ-C30 and BR-23.[38] We attribute this to the different cultural contexts in which the scales were developed. The Cuban instrument has nine PsF items and the SR domain includes three items about relations with family, spouse and friends, whose formulation in the way you need it was influenced by patient focus groups.

Another difference appears with location of the item sleeping disturbance. This was associated with emotional stress by Cuban patients, but not by the EORTC QLQ-C30, which classified it as a symptom.[38] In a study that interviewed 75 breast cancer patients treated with surgery and chemotherapy to compare a Cuban questionnaire to FACT-B, an international questionnaire specific for breast cancer,[23,39] researchers found that Cuban women needed additional explanation to understand the FACT$B$ items. The items in the Cuban-developed questionnaire were expressed more simply. Researchers reported that on being asked some questions from FACT-B ( I am losing hope in my fight against this disease, I worry about dying, I worry about my disease getting worse) some patients were silent for a few seconds, looking at the interviewer, as if surprised by the question, and a few burst into tears. Such reactions were not seen with the other FACT-B items, nor with any in the Cuban instrument.[40]

The role of reliability and validity evaluation in such instruments is fundamentally to collect evidence for their improvement. Since it was possible to evaluate the revised version using the same database, we were able to evaluate CV-MRT-01 without needing to interview more patients. Item formulation and measurement intervals were identical; only the number of items and their location in the synthetic variables changed. We inferred that since response burden was negligible with the 42-item CV-MRT-P, there would be no greater response burden for the 33-item CV-MRT-01.

Internal consistency assessment of both versions indicated an increase in homogeneity in the revised version, with the elimination of items that correlated poorly with their domains or the overall score. The first version already had acceptable internal consistency: Cronbach alpha $>0.8$ indicated that the scale measures different aspects of the same construct. Test-retest reliability was also acceptable, with repetitive results when applied to patients whose disease remained stable.[41]

Construct validity showed that, in both versions, the strongest and weakest correlations matched well with predictions (convergent and divergent validity). However, some items in the adverse 
effects domain did not present the expected patterns of convergence. These results are typical of clinimetric domains, since neither symptoms nor adverse effects are indicators per se of HRQOL; rather, they cause changes in level. Thus, it is not surprising that causal items show weak correlations with their own domain, or stronger correlations with another domain to which they do not theoretically belong. Such atypical behavior is the reason why internal consistency, as well as convergent and divergent validity, are not considered primordial properties of instruments that include causal items.

EFA was useful in simplifying the instrument and making it a more efficient measure of HRQOL. The 33 items retained explain the highest percentage of covariation possible and minimize information loss. This result needs to be corroborated in the future with confirmatory factorial analysis. EFA is not the most suitable analytic tool for instruments that include causal variables, because different data sets can have different factorial structures; e.g., hair loss and nausea and vomiting would correlate strongly in a study of patients on chemotherapy, but weakly in patients on hormonal therapy, which does not have the same adverse effects.[42]

Discriminant validity was satisfactory. CV-MRT-01 detected different levels of HRQOL between subgroups of clinical stages expected to have different scores. Such comparisons between groups known to differ are one way to evaluate discriminatory capacity. In the particular case of breast cancer patients, it was useful to verify that, in a period common to all patients (second measurement), the radiotoxic burden of radiotherapy differed among patients at different clinical stages of the disease. The importance of discriminant validity for an instrument with clinimetric variables is its ability to identify individuals with different clinical variables in cross-sectional designs.

The large- and medium-HRQOL effects detected by CV-MRT-01 (both domains and discrete items) are plausible. The before/after (radiotherapy) change reflects deterioration in HRQOL due to radiotherapy and the after/4-weeks-after change reflects a degree of recovery from its effects. Our findings are consistent with those of Manzanec's study of radiotherapy's negative effects on HRQOL.[8] The ability of an instrument to detect HRQOL changes at different clinical stages is critically important in clinical trials. Predictive validity is a particularly desirable property for scales that include causal items, because of ability to detect prospective clinical changes over time.[43]

Clinical interpretability is another important characteristic for this kind of instrument. In this respect, coherence was observed between the clinical phenomena measured and instrument scores. Scores for the synthetic variables fell within the milddeterioration category. The values for two of these, PhF and PsF, rose in a straight line over the three measurements. Both $A E$ and $S R$ were lower at the end of the treatment period but recovered somewhat in the final measurement. The two discrete items, perceived general health and perceived HRQOL, changed little over the three measurements, but did move from the upper moderate category to mild deterioration four weeks after treatment ended.

This study confirms the validity and reliability CV-MRT-01. However, as Guyatt notes, validation should not be an all-or-nothing project.[44] Although our initial assumptions have been borne out, we will continue to evaluate the instrument to test its validity in different studies.

A limitation of this study is that the instrument was developed and validated with a highly selected patient population: those referred for treatment at INOR, a tertiary-level institution. Even though the patients came from different regions of Cuba, they do not constitute a nationally representative sample, restricting generalizability.

\section{CONCLUSIONS}

CV-MRT-01 demonstrates reasonably well the properties required for measurement of HRQOL in Cuba among breast cancer patients receiving radiotherapy, particularly those most pertinent to causal variables (reliability, predictive validity and interpretability). The study supports CV-MRT-01's inclusion in clinical trials of radiotherapy in such Cuban patients.

\section{REFERENCES}

1. Welzel G, Boch A, Sperk E, Hofmann F, KrausTiefenbacher $U$, Gerhardt A, et al. Radiationrelated quality of life parameters after targeted intraoperative radiotherapy versus whole breast radiotherapy in patients with breast cancer: results from the randomized phase III trial TARGIT-A. Radiat Oncol [Internet]. 2013 Jan 7 [cited 2014 Jul 24];8:9. Available from: http://www.ncbi .nlm.nih.gov/pmc/articles/PMC3896671/

2. Lemieux J, Goodwin PJ, Bordeleau LJ, Lauzier $\mathrm{S}$, Théberge V. Quality-of-life measurement in randomized clinical trials in breast cancer: an updated systematic review (2001-2009). J Natl Cancer Inst. 2011 Feb 2;103(3):178-231.

3. Bridou M, Aguerre C. Specificities, causes and consequences of cancer anxiety: a review of behavioural and cognitive approach contributions. Psycho-Oncol [Internet]. 2010 Dec [cited 2014 Jul 16];4(1 Suppl):S26-32. Available from: http:// link.springer.com/article/10.1007\%2Fs 11839 -010-0250-x\#page-1. French.

4. Darrow SM, Follete WC. Starting a functional assessment of depression (FAD): development of FAD. Behav Modif. 2011 Mar;35(2):201-29.

5. Bossola M, Murri R, Onder G, Turriziani A, Fantoni M, Padua L. Physicians' knowledge of health-related quality of life and perception of its importance in daily clinical practice. Health Qual Life Outcomes [Internet]. 2010 Apr 23 [cited 2013 Dec 20];8:43. Available from: http://www.biomed central.com/content/pdf/1477-7525-8-43.pdf

6. Lai BP, Tang CS, Chung TK. A prospective longitudinal study investigating neuroticism and mastery as predictors of quality of life among Chinese gynecologic cancer survivors. Qual Life Res [Internet]. 2010 Sep [cited 2013 Dec 20];19(7):931-41. Available from: http://link.springer.com/article/ 10.1007\%2Fs11136-010-9657-x

7. Yang EJ, Shin EK, Shim HI, Lim JY. Psychometric properties of scale constructed from the international Classification of Functioning, Disability and Health (ICF) core set for breast cancer based on Rasch analysis. Support Care Cancer [Internet]. 2014 May 13 [cited 2014 Jul 24]. Available from: http://link.springer.com/art\%253A10.1 007\%252Fs00520-014-2277-1.pdf

8. Mazanec SR, Daly BJ, Douglas S, Musil C. Predictors of psychosocial adjustment during the postradiation treatment transition. Western $\mathrm{J}$ Nursing Res. 2011 Jun;33(4):540-59.

9. Waller A, Forshaw K, Bryant J, Mair S. Interventions for preparing patients for chemotherapy and radiotherapy: A systematic review. Support Care Cancer. 2014 Aug [cited 2014 Jul 24];(8):22972308. Available from: http://link.springer.com/art icle/10.1007\%2Fs00520-014-2303-3

10. Delgado-Sanz MC, García-Mendizábal MJ, Pollán M, Forjaz MJ, López-Abente G, Aragonés $\mathrm{N}$, et al. Heath-related quality of life in Spanish breast cancer patients: a systematic review. Health Qual Life Outcomes [Internet]. 2011 Jan 14 [cited 2014 Feb 5];9:3. Available from: http:// www.hqlo.com/content/9/1/3

11. Montazeri A. Health-related quality of life in breast cancer patients: A bibliographic review of the literature from 1974 to 2007. J Exp Clin Cancer Res [Internet]. 2008 Aug 29 [cited 2013 Dec 20];27:32. Available from: http://www.ncbi.nlm .nih.gov/pmc/articles/pmid/18759983/

12. Salas Zapata C, Grisales Romero H. Calidad de vida y factores asociados en mujeres con cáncer de mama en Antioquia, Colombia. Rev Panam Salud Pública [Internet]. 2010 Jul [cited 2013 Dec 20];28(1):9-18. Available from: www .scielosp.org/pdf/rpsp/v28n1/v28n1a02.pdf. Spanish.

13. Spitzer WO, Dobson AJ, Hall J, Chesterman E, Levi J, Shepherd R. Measuring the qual- 
ity of life of cancer patient. J Chronic Dis. 1981;34(12):585-97.

14. Barofsky I. Why perform a quality or quality of life assessment? Qual Life Res [Internet]. 2012 May [cited 2014 Jul 24];21(4):633-6. Available from: http://link.springer.com/ article/10.1007\%2Fs11136-011-9962-z

15. Lai JS, García SF, Salsman JM, Rosenbloom S, Cella D. The psychosocial impact of cancer: evidence in support of independent general positive and negative components. Qual Life Res [Internet]. 2011 Jun 4 [cited 2014 Jul 24]. Available from: http://link.springer.com/article/10.1007\%2 Fs11136-011-9935-2

16. Meldahl ML, Acaster S, Hayes RP. Exploration of oncologists' attitudes toward and perceived value of patient-reported outcomes. Qual Life Res [Internet]. 2013 May [cited 2014 Jul 24];22(4):725-31. Available from: http://link.springer.com/article/10 .1007\%2Fs 11136-012-0209-4

17. Bentley JP, Brown CJ, McGwin G Jr, Sawyer $P$, Allman RM, Roth DL. Functional status, lifespace mobility, and quality of life: a longitudinal mediation analysis. Qual Life Res [Internet]. 2013 Sep [cited 2014 Jul 24];22(7):1621-32. Available from: http://link.springer.com/article/10.1007\%2 Fs11136-012-0315-3

18. Chopra I, Kamal K. A systematic review of quality of life instruments in long-term breast cancer survivors. Health Qual Life Outcomes [Internet]. 2012 Jan [cited 2014 Jul 24]. Available from: http://www.ncbi.nlm.nih.gov/pmc/articles/ PMC3280928/

19. Salsman JM, Lai JS, Hendrie HC, Butt Z, Zill $\mathrm{N}$, Pilkonis PA, et al. Assessing psychological well-being: self-report instruments for the $\mathrm{NIH}$ Toolbox. Qual Life Res [Internet]. 2014 Feb [cited 2014 Jul 24];23(1):205-15. Available from: http://link.springer.com/article/10.1007\%2 Fs11136-013-0452-3

20. Reeve BB, Wyrwich KW, Wu AW, Velikova G, Terwee CB, Snyder CF, et al. ISOQOL recommends minimum standards for patient-reported outcome measures used in patient-centered outcomes and comparative effectiveness research. Qual Life Res [Internet]. 2013 Oct [cited 2014 Jul 24];22(8):1889-905. Available from: http://link .springer.com/article/10.1007\%2Fs11136-012 $-0344-y$

21. Siegrist J. Social determinants of health-a cross cultural perspective. Int J Public Health. 2008;53(6):277-8.

22. Grossi E, Luigi P, Blessi GT, Cerutti R. The impact of culture on the individual subjective well-being of the Italian population: an exploratory study. Applied Res Qual Life. 2011;6(4):387-410.

23. Cella DF, Tulsky DS, Gray G, Sarafian B, Linn E, Bonomi A, et al. The Functional Assessment of Cancer Therapy (FACT-G) scale: development and validation of the general measure. J Clin Oncol. 1993 Mar;11(3):570-9.

24. Aaronson NK, Ahmedzai S, Bergman B, Bullinger M, Cull A, Duez NJ, et al. The European Organization for Research and Treatment of Cancer QLQ-C30: a quality-of-life instrument for use in international clinical trials in oncology. J Natl Cancer Inst. 1993 Mar 3;85(5):365-76.

25. El Fakir S, Abda N, Bendahhou K, Zidouh A, Bennani $M$, Errihani $H$, et al. The European organization for research and treatment of cancer quality of life questionnaire-BR23 breast cancer-specific quality of life questionnaire: psychometric properties in a Moroccan sample of breast cancer patients. BMC Research Notes [Internet]. 2014 Jan 21 [cited 2014 Jul];7:53. Available from: http://www.biomedcentral.com/1756-0500/7/53

26. Sánchez R, Ballesteros M, Arnold BJ. Validation of the FACT-G scale for evaluating quality of life in cancer patients in Colombia. Qual Life Res [Internet]. 2011 Feb [cited 2014 Feb 18];20(1):19-29. Available from: http://dx.doi.org/10.1007/s11136 -010-9727-0

27. Fayer PM, Hand DJ. Causal variables, indicator variables and measurement scales: an example from quality of life. J Royal Stats Soc [Internet]. 2002 Jun 19 [cited 2010 Feb 18];165(2):233-53. Available from: http://onlinelibrary.wiley.com/ doi/10.1111/1467-985X.02020/abstract

28. Boehmer S, Luszczynska A. Two kinds of items in quality of life instruments: 'indicator and causal variables' in the EORTC qlq-c30. Qual Life Res. 2006 Feb:15(1):131-41.

29. Arias MM, Giraldo CV. El rigor científico en la investigación cualitativa. Invest Educ Enferm. 2011;29(3):500-14. Spanish.

30. Arrarás $\mathrm{Jl}$, Arias $\mathrm{F}$, Illarramendi $\mathrm{JJ}$, Manterola ES, Salgado E, Domínguez MA, et al. Calidad de vida relacionada con la salud en los servicios de Oncología del Hospital de Navarra. Grupo de Calidad de Vida de la EORTC. An Sist Sanit Navar 2011 Jan-Apr ;34(1):9-20. Spanish.

31. Morales P, Urosa SB, Blanco BA. Construcción de escalas de actitudes tipo Likert. Una guía práctica. 2nd ed revised. Madrid: Editorial La Muralla; 2003. 175 p. Spanish.

32. García JE, Gil FJ, Rodríguez GG. Análisis Factorial. Madrid: Editorial La Muralla, S.A; 2000.

33. Fayer PM. Scores and Measurements: Validity, Reliability, Sensitivity Quality of Life Assessment. In: Fayer PM, Machin D, editors. Quality of Life: The Assessment, Analysis and Interpretation of Patient-reported Outcomes. 1st ed. New York: John Wiley \& Sons; 2001. $404 p$

34. Cronbach LJ, Gleser GC, Nanda H, Rajaratnam $\mathrm{N}$. The dependability of behavioral measurements: Theory of generalizability for scores and profiles. New York: John Wiley \& Sons; 1972 Jun 14. $410 \mathrm{p}$.

35. Feise RJ, Michael Menke J. Function rating index: a new valid reliable instrument to measure the magnitude of clinical change in spinal conditions. Spine (Phila Pa 1976). 2001 Jan 1;26(1):78-86

36. Cohen J. Statistical Power Analysis for the Behavioral Sciences. New York: Academic Press; 1977. $474 \mathrm{p}$.

37. Fayer PM. Clinical Interpretation Quality of Life Assessment, Analysis and Interpretation. In: Fayer PM, Machin D, editors. Quality of Life: The Assessment, Analysis and Interpretation of Patient-reported Outcomes. 1st ed. New York: John Wiley \& Sons; 2001. $404 p$

38. Kim EJ, Ko SK, Kang HY. Mapping the cancer-specific EORTC QLQ-C30 and EORTC QLQ-BR23 to the generic EQ-5D in metastatic breast cancer patients. Qual Life Res. 2012 Sep;21(7):1193-203.

39. Fallowfield LJ, Leaity SK, Howell A, Benson S, Cella D. Assessment of quality of life in women undergoing hormonal therapy for breast cancer: validation of an endocrine symptom subscale for the FACT-B. Breast Cancer Res Treat. 1999 May:55(2):189-99.

40. González FY. Validación del cuestionario (CVJF) para evaluar calidad de vida de los pacientes con cáncer de mama [thesis]. [Havana]: National School of Public Health (CU); 1999. Spanish.

41. Streiner DL, Norman GR. Health Measurement Scales. A practical guide to their development and use. 4th ed. Oxford: Oxford University Press; 2008 Dec. 450 p.

42. Fayer PM. Factorial Analysis. In: Fayer PM, Machin D, editors. Quality of Life: The Assessment, Analysis and Interpretation of Patientreported Outcomes. 1st ed. New York: John Wiley \& Sons; 2001. 404 p.

43. Fayer PM. Principles of Measurement Scales. In: Fayer PM, Machin D, editors. Quality of Life: The Assessment, Analysis and Interpretation of Patient-reported Outcomes. 1st ed. New York: John Wiley \& Sons; 2001. $404 p$.

44. Guyatt GH, Feeny DH, Patrick DL. Measuring Health-related QoL. Ann Intern Med. 1993 Apr 15;118(8):622-9.

\section{THE AUTHORS}

Josefina Lugo Alonso (Corresponding author: jlainor@infomed.sld.cu), physician specializing in biostatistics. Associate professor, clinical research department, Oncology and Radiobiology Institute (INOR), Havana, Cuba.

Misleidy Nápoles Morales, oncologist with a master's degree in comprehensive women's health. Assistant professor, radiotherapy service, INOR, Havana, Cuba.

Inés Pérez Braojo, oncologist. Associate professor, breast health service, INOR, Havana, Cuba.

Niurka Ordaz Hernández, nurse. Assistant professor, radiotherapy service, INOR, Havana, Cuba.

Mario Luzardo Verde, mathematician with a doctorate in behavioral sciences. Professor, Psychology Faculty, University of the Republic of Uruguay, Montevideo, Uruguay.

Leticia Fernández Garrote, biostatistician with a doctorate in medical sciences. Full professor and researcher, National School of Public Health, Havana, Cuba.

Submitted: April 2, 2014

Approved for publication: July 21, 2014 Disclosures: None 